\title{
An open Web-based system for the analysis and sharing of animal tracking data
}

\author{
Ross G Dwyer ${ }^{1 *}$, Charles Brooking ${ }^{2}$, Wilfred Brimblecombe ${ }^{2}$, Hamish A Campbell ${ }^{1,4}$, Jane Hunter ${ }^{2}$, \\ Matthew Watts ${ }^{3}$ and Craig E Franklin ${ }^{1}$
}

\begin{abstract}
Background: Improvements in telemetry technology are allowing us to monitor animal movements with increasing accuracy, precision and frequency. The increased complexity of the data collections, however, demands additional software and programming skills to process, store and disseminate the datasets. Recent focus on data availability has also heightened the need for sustainable data management solutions to ensure data integrity and provide longer term access. In the last ten years, a number of online facilities have been developed for the archiving, processing and sharing of telemetry data. These facilities offer secure storage, multi-user support and analysis tools and are a step along the way to improving data access, long-term data preservation and science communication. While these software platforms promote data sharing, access to the majority of the data and to the software behind these systems remains restricted. In this paper, we present a comprehensive, highly accessible and fully transparent software facility for animal movement data.
\end{abstract}

Results: The online system we developed (http://oztrack.org) offers a set of robust, up-to-date and accessible tools for managing, processing, visualising and analysing animal location data and linking these outputs with environmental datasets. As OzTrack uses exclusively free and open-source software, and the source code is available online, the system promotes open access not only to data but also to the tools and software underpinning the system.

Conclusions: We outline the capabilities and limitations of the infrastructure design and discuss the uptake of this platform by the Australasian biotelemetry community. We discuss whether an open approach to analysis tools and software encourages a more open approach to sharing data, information and knowledge. Finally, we discuss why a free and open approach enhances longer term sustainability and enables data storage facilities to evolve in parallel with the telemetry devices themselves.

Keywords: Electronic tagging, Electronic tags, Environmental data, GPS, Information management, Kalman filter, Movement ecology, Open access, OzTrack, PostgreSQL, PostGIS, R, Relational database, Tracking software, VHF, Wildlife monitoring

\section{Background}

Animal biotelemetry is a dynamic and rapidly evolving field of ecology. The number of publications featuring the use of telemetric devices to provide fundamental insights into a species' ecology has increased steadily over the past 30 years $[1,2]$. Technological advancement during this time has improved positional accuracy and is allowing the collection of more frequent location fixes over longer time periods [3,4]. Furthermore, tag size and weight have substantially reduced [5], as has unit cost

\footnotetext{
*Correspondence: ross.dwyer@uq.edu.au

${ }^{1}$ EcoLab, School of Biological Sciences, The University of Queensland,

Brisbane, Australia

Full list of author information is available at the end of the article
}

[6], broadening the spectrum of species and life stages that can be studied. These developments in tracking technology and data acquisition have reduced the effort required to gather high-quality animal location data, providing researchers with an opportunity to build a coherent picture of how animals behave within their natural environment.

The relative ease and low cost by which temporally dense sets of animal locations can now be collected is increasing the volume of data collected and thus making effective management of the data challenging. Telemetry devices now routinely generate datasets that are larger than off-the-shelf software tools or spreadsheet applications can handle $[7,8]$. While dedicated, proprietary 
software platforms for processing these tracking data are available directly from the tag manufacturers, many largescale and long-term studies utilise tags sourced from multiple manufacturers (e.g. $[9,10])$. In this scenario, a number of independent applications may be required; these include software tools designed for spatial data management and visualisation, as well as tools to filter and analyse spatiotemporal datasets. Typically, this requires data replication and export/import procedures across multiple software applications, which can be time-consuming and prone to error and duplication [11].

To ensure data persistence and to facilitate comparative studies across species and localities, the secure, consistent and efficient management of data is essential. If raw data are stored locally in personal databases, they are at risk of being lost or discarded due to disk failure or the data owner changing offices or careers. As data are collected as a result of disturbing animals, and many programs are funded by public money, there is a responsibility for the researcher to provide public access to their data once funding and publishing obligations have been met $[12,13]$. Data publishing is already a high priority in many fields, and many research journals and funding bodies now require that data supporting the results of studies are archived in an appropriate public archive which guarantees their re-use and preservation [14].

While some researchers may be unwilling to transfer their datasets into an online data repository (e.g. for fear of others misusing their data or using it without proper acknowledgement [15]), data management and dissemination through the Internet offers some clear advantages. For example, ongoing telemetric studies may require updating on a regular basis with new data and recent deployments [16], and project collaborators may want to be kept informed on the study's progress and the current whereabouts of tagged animals. The data may be useful for educational or public outreach purposes, which can increase the profile of the research through mass media and articles in national and international news outlets [17]. Increased data transparency may also stimulate collaboration, through the sharing of data between research groups and specialists in the same or related fields. This increased synergy may reduce the need to collect further or new data and can lead to academic as well as financial gains $[14,18]$. While a number of groups have succeeded in developing their own infrastructure for this purpose (e.g. Whalenet [19], the Narragansett Bay Coyote Study [20], the Information System for the Analysis and Management of Ungulate Data [21]), developing such infrastructure requires considerable time and resources, including collaborative input from information technology (IT) and e-Science experts.

In recognition of these challenges, some wildlife biologists have stressed a need for free online facilities with standardised tools and techniques for telemetry data
$[11,17,22]$. These facilities should not be limited to data management but should also support the analysis, visualisation and sharing of animal tracking data. A number of Web-based animal telemetry data repositories are currently available for this purpose. These include Movebank [23], OBIS-SEAMAP (Ocean Biogeographic Information SystemSpatial Ecological Analysis of Megavertebrate Populations) [24], Wildlife Tracking (formerly Seaturtle.org) [17], Wireless Remote Animal Monitoring [25] and the European Roe Deer project [26]. Data within these repositories are stored in a way which permits multi-user support and data sharing, a secure and consistent storage system and analysis and filtering tools of varying complexity. However, the data policies of these facilities are still restrictive [13], with only a small fraction of contributed datasets accessible for downloading or viewing. Furthermore, the infrastructure underlying these repositories remains guarded (or focused on a single species), reducing the potential for the examination and further development of these tools by the wider biotelemetry community.

In this paper, we introduce an online platform based on a spatially enabled database management system (DBMS) for the archiving, analysis and dissemination of multi-species tracking data: OzTrack.org [27,28]. Our main objectives were twofold. The first objective was to develop a free, open source, highly accessible yet comprehensive Webbased application with automated procedures and tools for processing animal location data. Our second objective was to increase data and metadata visibility, thereby encouraging a data sharing culture amongst the animal tracking community. These objectives were achieved through a collaborative partnership between biologists and software engineers based at The University of Queensland, and a steering committee comprising researchers and practitioners of wildlife telemetry and eResearch services. Researchers conducting biotelemetry studies now have access to the tools and contributed telemetry datasets resulting from this collaboration. Here, we provide a description of the software architecture, discuss system uptake and illustrate system functionality using existing OzTrack projects. Finally, we discuss future developments and argue why an open-access approach to data, analysis tools and software is fundamental if we are to keep up with the rapid advancement of the devices themselves.

\section{Methods}

\section{The database management system}

The OzTrack system was implemented using the opensource object-relational database system PostgreSQL [29] (version 9.1), with its spatial extension PostGIS [30] (version 2.0). These tools were overlaid within a Java Web application and a user-friendly Web interface constructed to enable project creation and metadata upload (Figure 1). The input process is straightforward: project metadata is 


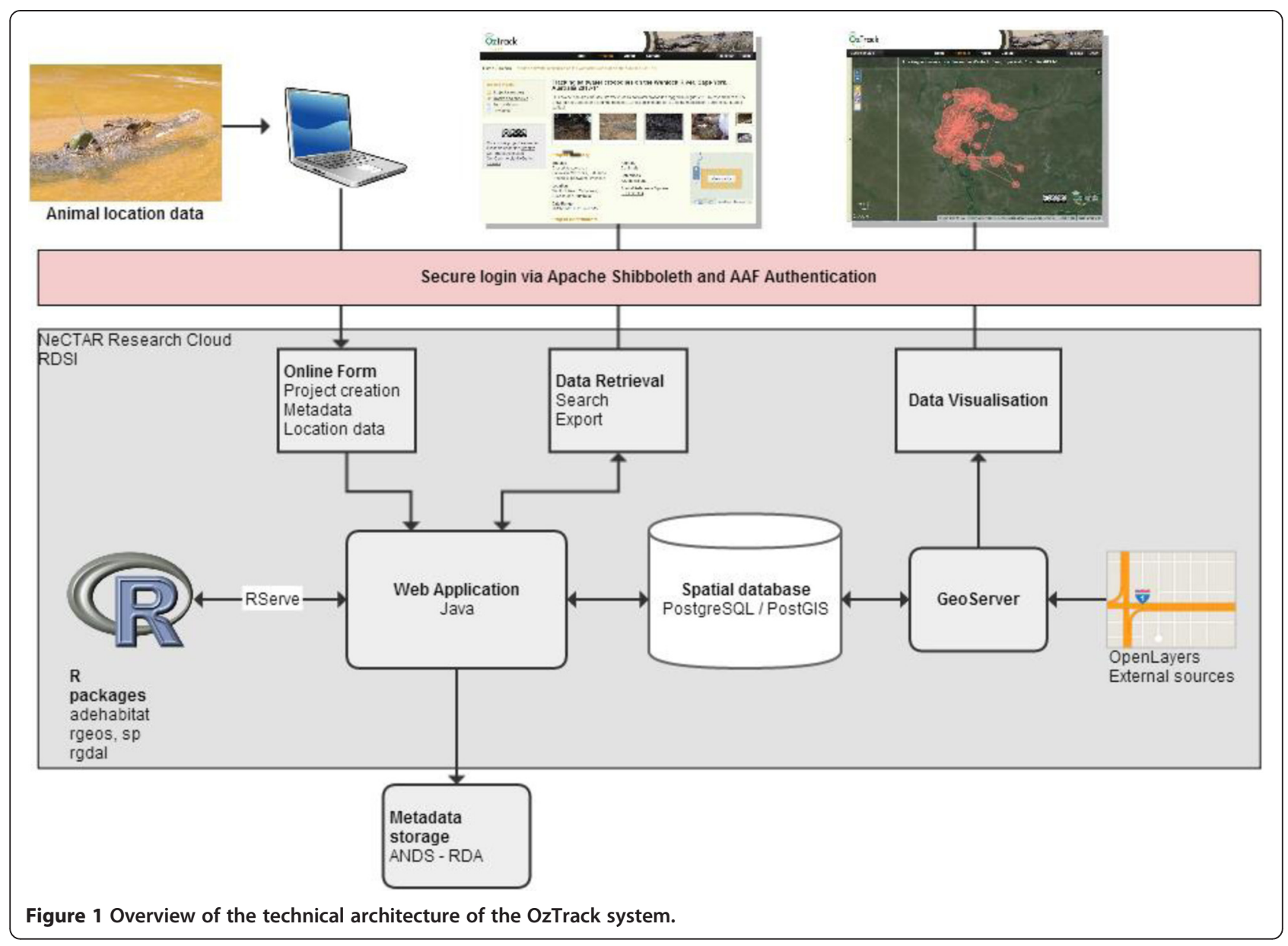

entered via an online form, with animal location data uploaded in Comma-Separated Value (.CSV) or Microsoft Excel (.XLS) files. To increase system flexibility, animal location datasets may be uploaded with dates and times in the format "dd/mm/yyyy HH:MM" or "yyyy-mm-dd HH: MM:SS" and geographical coordinates as decimal degrees, degrees decimal minutes or degrees minutes seconds in the World Geodetic System (WGS) 1984 datum. The entry of a species' common name and species' scientific name is automated using the Atlas of Living Australia Autocomplete Web service [31]. There is also the option for users to enter the species' common and scientific name manually, increasing the transferability to study outside of Australia. Metadata describing data collections, users and projects within the system are published to Australian National Data Service-Research Data Australia (ANDS-RDA) in the Registry Interchange Format-Collections and Services (RIF-CS) metadata format. The OzTrack system is hosted on server infrastructure within the National eResearch Collaboration Tools and Resources (NeCTAR) Research Cloud. This greatly reduced the administration costs and effort compared with hosting the system locally.

\section{Mapping functionality}

Interactive mapping and visualisation services were obtained from the open-source GeoServer software server [32], providing a consistent interface for rendering maps and feature data. Data are visualised via an in-browser OpenLayers map, which includes Google Maps base imagery, and measure and zoom functionality (Figure 1). Climatic, environmental and political spatial layers were obtained from online remote sensing repositories covering both the terrestrial and the marine environments (Additional file 1: Table S1). Layers are rendered as raster images on the server side using GeoServer, which are then visualised as Portable Network Graphics (.PNG) image tiles at the required zoom level. This feature was found to dramatically speed up the processing time, particularly for datasets containing large numbers of location fixes (e.g. those approaching 10,000 detections) [27]. Requests for map tiles, feature attributes and map legends were developed using GeoServer's WMS interface. Once visualised, Structured Query Language (SQL) statements are used to query the animal locations, trajectories and spatial layers. Full details on the implementation of these tools, and the 
underlying code, can be found on the OzTrack GitHub website [33].

\section{Analysis tools}

Once location data are uploaded into a project, movement metrics such as the total number of fixes per deployment, the mean number of fixes per day, animal step length and the minimum distance travelled are extracted automatically using a series of SQL statements. Standard filtering options for data visualisation and analysis were implemented using update statements in SQL, such as setting a start and end date or tag location quality (i.e. Argos location class, dilution of precision class). OzTrack also provides a polygon selection tool permitting the user to manually delete improbable location estimates (e.g. an inland location for a marine species) from a project. To perform more complex spatial analyses and data filtering procedures, the system connects to the $\mathrm{R}$ programming environment [34] via RServe [35]. Spatial analysis and filtering tools contained within contributed R packages (Table 1, Additional file 2: Table S2), and our own customised R code and R-Forge (a R interface to GDAL, OGR and PROJ.4) are all called inside the OzTrack system as native procedures (Figure 1). Support regarding the parameterisation of the analysis and filter tools can be found in the form of help icons positioned next to the appropriate tool or through links provided to the $\mathrm{R}$ package help files, vignettes and publications via the system's Web portal [33]. Spatial distances are calculated as great circle distances on the WGS 1984 ellipsoid, and area calculations are performed after locations are converted from this geographical coordinate system into a suitable projected spatial reference system (SRS). SRS conversions are conducted using PROJ.4 projection arguments in the rgdal $\mathrm{R}$ package [36], with EPSG codes obtained from spatialreference.org [37]. The results of these procedures (i.e. vector-based objects: points, lines or polygons) are returned to OzTrack using the Keyhole Markup Language (KML) format via RServe for visualisation.

All capture and tagging procedures were carried out with approval from The University of Queensland Animal Ethics Committee (Cassowaries:SIB/935/08/EPA ; Crocodiles: SBS/204/11/ARC/AUST ZOO; Koalas: CMLR/091/

Table 1 Current tool functionality and data formats for export

\begin{tabular}{|c|c|c|}
\hline Tool & Description & Exportable format \\
\hline \multicolumn{3}{|l|}{ Editing tools } \\
\hline Polygon selection & Manual selection of locations for removal by drawing polygons. & $. \mathrm{CSV}, . \mathrm{KML}, \mathrm{SHP}$ \\
\hline Date filter & Filter locations by date range. & $. C S V, . K M L, . S H P$ \\
\hline Location class filter & Locations with a lower location accuracy than the minimum specified will be deleted. & $. C S V, . K M L, . S H P$ \\
\hline Speed filter & Locations that imply the animal has exceeded the specified maximum speed will be deleted. & $. \mathrm{CSV}, . \mathrm{KML}, \mathrm{SHP}$ \\
\hline Kalman filter & $\begin{array}{l}\text { This filter applies a state-space model combined with a Kalman filter to the location data to } \\
\text { predict the "most probable" track. }\end{array}$ & $. \mathrm{CSV}, . \mathrm{KML}, \mathrm{SHP}$ \\
\hline Kalman filtered data (SST) & $\begin{array}{l}\text { This filter applies a state-space model combined with a Kalman filter to the location and } \\
\text { temperature sensor data predict the 'most probable' track. The tag-recorded SST is matched } \\
\text { with external SST data collected by the National Oceanic and Atmospheric Administration. }\end{array}$ & $. \mathrm{CSV}, . \mathrm{KML}, . \mathrm{SHP}$ \\
\hline \multicolumn{3}{|l|}{ Analysis tools } \\
\hline Movement metrics & $\begin{array}{l}\text { Track distance, mean step length and mean step speed are extracted using the great circle } \\
\text { distance algorithm. }\end{array}$ & - \\
\hline Minimum convex polygon & A home range measure which uses the smallest area convex set that contains the location data. &.$K M L$, SHP \\
\hline Kernel utilisation distribution & $\begin{array}{l}\text { A home range measure which uses the utilisation distribution to estimate the probability } \\
\text { that an animal will be found at a specific geographical location. }\end{array}$ &.$K M L$, SHP \\
\hline Kernel Brownian bridge & $\begin{array}{l}\text { A home range measure which uses the utilisation distribution to estimate the probability } \\
\text { that an animal will be found at a specific geographical location. Unlike the fixed kernel } \\
\text { approach, the Kernel Brownian Bridge incorporates serial autocorrelation between fixes into } \\
\text { the estimate. }\end{array}$ & $. K M L, . S H P$ \\
\hline Alpha hull & $\begin{array}{l}\text { A generalisation of the convex hull, which uses Delaunay triangulation to objectively crop } \\
\text { low use areas from a polygon surface. }\end{array}$ & $. K M L, . S H P$ \\
\hline Local convex hull & $\begin{array}{l}\text { A home range measure which creates utilisation distributions based on the local nearest- } \\
\text { neighbour convex hulls. These are formed by constructing convex hulls around each location in } \\
\text { the animal's trajectory then jointing these hulls together, iteratively, to form isopleths. }\end{array}$ & $. K M L, . S H P$ \\
\hline Heat map & $\begin{array}{l}\text { This tool generates a grid over the study area and uses a coloured gradient to visually } \\
\text { identify areas of high usage by the tagged animal. Can be applied to either points or } \\
\text { connectivity lines between points. }\end{array}$ &.$K M L$, SHP \\
\hline
\end{tabular}


12/ARC/RIO TINTO) and a Queensland Government permits (Cassowaries: DERM 2011/02/01; Crocodiles: WISP05268508; Koalas: WISP05609708).

\section{Results and discussion \\ System uptake}

At the time of writing (February 2014), the Web site has recorded 3,298 site visits (comprising 4,603 page views) and 92 registered users (including academics, university students, agency scientists and environmental consultants). In total, 1,417 tracks comprising 2,050,812 position fixes from 55 different species have been uploaded, with location data gathered using telemetric devices (i.e.
Argos, GPS, VHF, light-based geolocations and underwater acoustic), or re-sightings of individually marked animals.

During its development, OzTrack.org was used productively by a number of research groups and organisations to address a range of wildlife management and conservation scenarios. This included facilitating the visualisation of movements and generating home range estimates for koalas (Phascolarctos cinereus) inhabiting a highly fragmented landscape in South East Queensland, Australia (Figure 2). Here, OzTrack enabled researchers to construct home ranges and identify cleared areas where koalas fitted with GPS collars regularly crossed between habitat fragments. The findings from this study were used to focus habitat restoration activities on

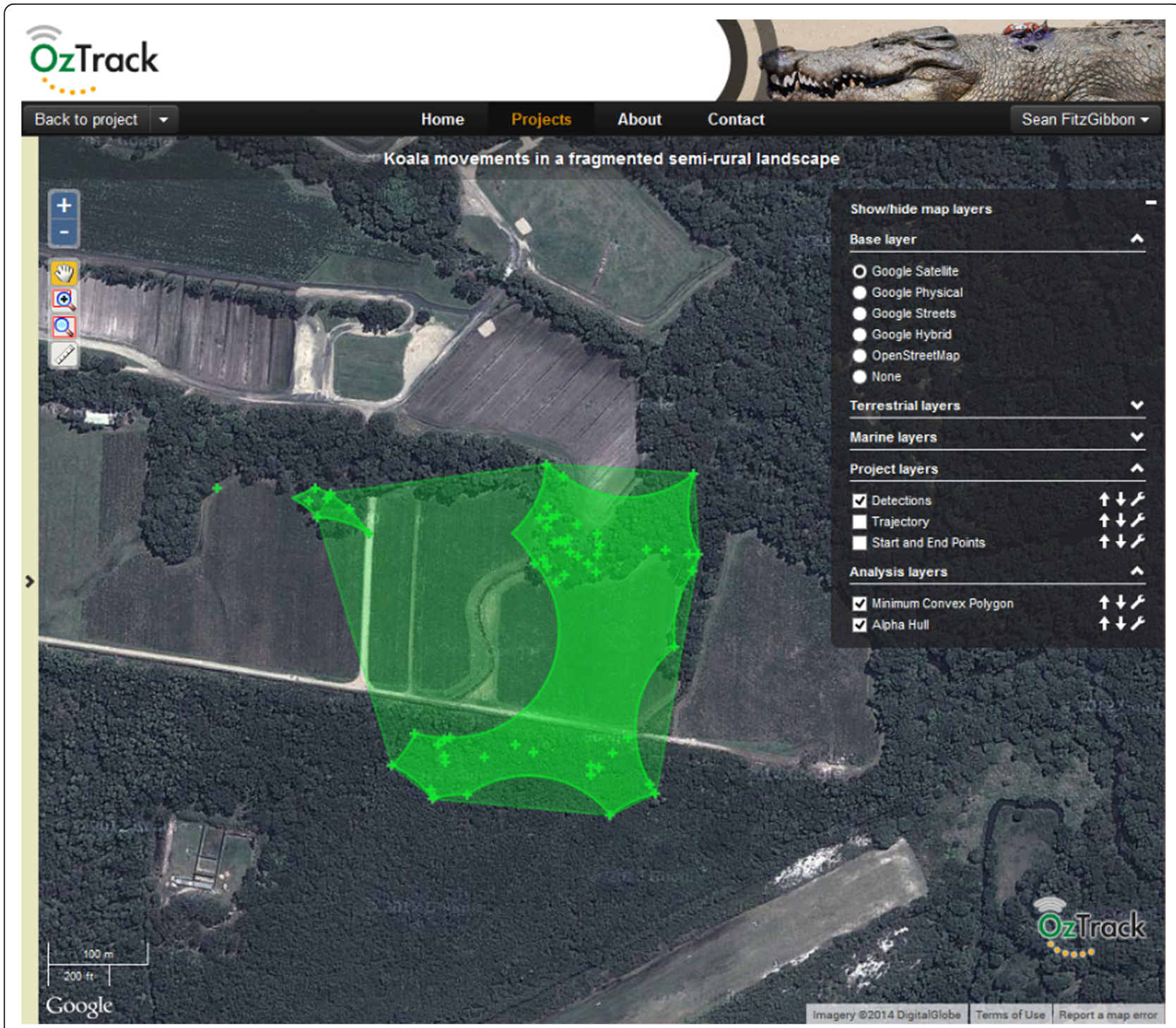

Figure 2 The locations and home range estimates of a GPS-collared koala in a highly fragmented landscape. Home ranges estimated using minimum convex polygon (MCP) and alpha hull methods. The locations are projected onto a Google satellite base layer and visualised within the OzTrack system portal. 


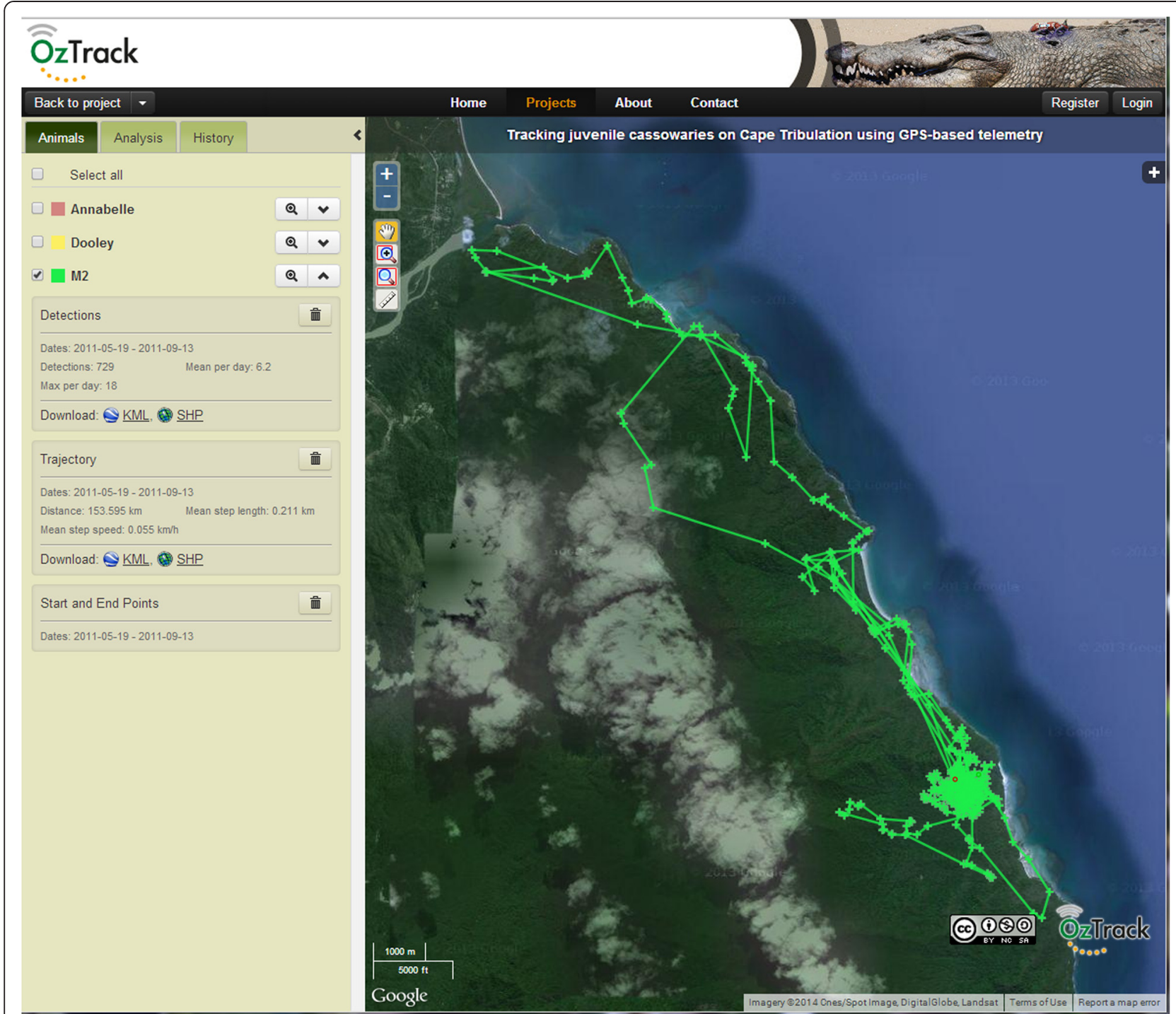

Figure 3 The track of a rehabilitated southern cassowary following release into the Daintree National Park. The information in the left panel provides metrics on the number of detections (locations) for that animal, the start and end date of the tracking period and the length of the animal's trajectory. The track of animal "M2" contains 729 GPS locations gathered between 19/05/2011 and 13/09/2011.

improving connectivity of koala habitat. OzTrack was also employed to monitor the success of a rehabilitation and release scheme for southern cassowaries (Casuarius casuarius) [38]. OzTrack's configurable permission tool allowed the raw locations, movement metrics and home range estimates generated from GPS-tagged birds to be shared between project collaborators (Figure 3). Those collaborators working in the study area were also able to provide valuable insight into local disturbances that helped explain the timing and locality of observed movement behaviours. By facilitating data discussion and multi-user support, OzTrack helped elucidate behaviours which would have otherwise been difficult to interpret. Finally, between September 2013 and February 2014, OzTrack was used as both a research tool and as a platform to promote public awareness towards estuarine crocodiles (Crocodylus porosus). Large crocodiles pose a significant risk to humans, and there are significant societal and political challenges in managing this potentially dangerous species [39]. To help raise public awareness regarding the habits of wild estuarine crocodiles, OzTrack was used to archive the movements of six adult crocodiles fitted with GPS transmitters (Figure 4). The project was open to the public to view the tracks in near real-time and apply a selection of analysis tools, and it was promoted via articles in the popular press and through public lectures. Promoting awareness through public outreach is necessary in order to explain science to the public and media; it is also a necessity to endorse fundraising activities, conservation measures and management decisions. 


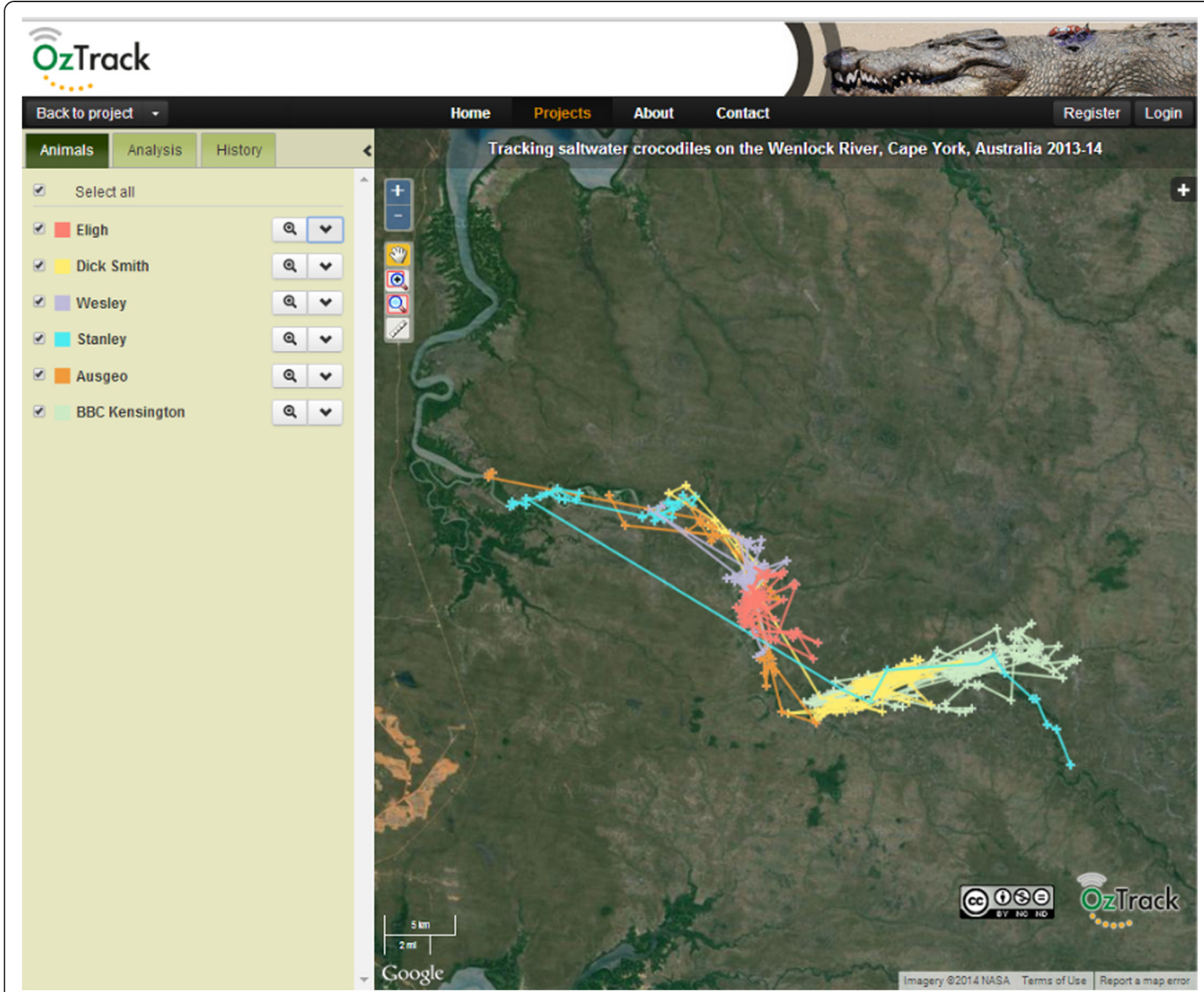

Figure 4 The movements of six estuarine crocodiles using Argos-linked GPS tags. The study was conducted in the Wenlock River in Cape York, Queensland, Australia. The colours of the points and lines correspond to an individual crocodile. This project open to the public and the locations of animals were updated on a regular basis for 6 months following the attachment of tags in September 2013.

\section{Open tools and software}

By running a spatial DBMS within research cloud infrastructure, we achieved a scalable, persistent and secure data storage capability in which large tracking datasets can be managed in a structured and consistent manner [27]. The system is able to visualise tracks and extract movement metrics from datasets containing hundreds of thousands of animal locations. Dynamic upload capabilities with automated data recognition allow location data to be ingested into the DBMS with minimal pre-processing prior to upload, regardless of origin, time zone or geographical region. Integrating tools for spatial data visualisation (GeoServer) [32] and analysis ( $R$ ) [34] combine the data archiving and processing components within a single online software platform [27]. As a result, location data can be edited, filtered, analysed and visualised with environmental and geopolitical layers without the need to convert and upload data files into separate software applications. This reduces the need for multiple copies of filtered and unfiltered datasets in numerous data formats (e. g. .TXT, .CSV and .SHP) and thus the potential for data duplication and error propagation.

To maximise software sustainability, the system was developed using exclusively open-source software components that ensure it can be open, interoperable, scalable and freely available. No additional software, add-on packages or plugins are required to run, visualise or export the raw, filtered or analysed data other than the Web browser (e.g. Internet Explorer, Firefox and Chrome). This approach offers some clear benefits over alternative applications which typically require a user fee or are dependent on downloading and installing commercial or non-commercial 
software. First of all, it is cost-effective, an important factor for smaller research bodies, consultancies and conservation organisations who have limited resources to dedicate towards software purchasing [40]. Second, the system is accessible from any computer or tablet with a Web browser. This benefits researchers who may work across multiple computers and devices and students or employees who may not have local administrative access on their computer. Third, the system provides a real-time collaborative environment where multiple users can access the same project. This is done via configurable permissions and access rights which are provided by the data owner to other OzTrack users through system's Web portal (Figure 1, [27]). This combination of free and open tools, infrastructure and a collaborative environment has been highlighted as key requirements to lead to more informed conclusions and management decisions $[5,41]$.

Our trials with tertiary students illustrated the system's potential for teaching how biotelemetry can be a useful research tool in wildlife ecology and conservation. The main advantage over conventional methods was that once informed of the underlying theory, users were able to carry out standard procedures for analysing animal telemetry data without any "entrance barriers" [40], such as the requirement for students to be already proficient in a geographical information system (GIS) or have prior experience in using R. Many of the analysis and filtering tools implemented within the OzTrack system were modified from original functions contained within contributed $\mathrm{R}$ packages (Additional file 1: Table S1). Rather than R's command-line interface, the system provides an intuitive user interface that links to these functions though the Web portal. Users can access models, alter parameters, run analyses, visualise results and download data for postprocessing without the need to develop or enter $\mathrm{R}$ code. Online help documentation and links to relevant publications are provided alongside each analysis or filtering tool as are tips for running models and providing reliable results. By integrating these tools within an accessible Web-based system, users can quickly understand how the choice of filter, model and parameter values influences estimates of animal movement and space use. Provided there is a thorough understanding of the statistical assumptions, we argue that by improving access to current tools in animal movement, research will encourage their uptake by the tracking community (particularly those inexperienced in R), thereby improving the quality and appropriateness of the analyses run.

The system's source code (including the underlying $\mathrm{R}$ code) has been released publically [33], providing users worldwide with the opportunity to verify, customise and rebuild the OzTrack system locally. This open approach to software is contrary to the "black-box" approach offered by many existing proprietary platforms, but is the same model adopted by Tagbase [42], and the R Programing Language [24] which sits at the core of OzTrack's analysis tools. An open system controlled, developed and managed by the global animal tracking community increases the potential for online facilities like OzTrack to evolve in parallel with advances in biotelemetry research. This scalability is vital for software long-term persistence, allowing for continual implementation and integration of new and powerful analysis tools, not only by a specific project or institution but also by the global biotelemetry community.

\section{Open data}

Registered users of OzTrack are able to create and describe new projects, upload datasets into specific projects, add users to projects and specify access controls and embargo periods. Once raw location data have been uploaded into a project, project owners can choose to embargo their data for a period of up to 3 years. While providing instantaneous access to telemetry datasets can be beneficial, this period was chosen because it provides adequate time for data owners to fulfil funding and publishing obligations before public access is granted. Data are protected under a number of Creative Commons Attributions Licence agreements, ranging from fully open access with no copyright [43], to strict limitations on usage [44]. Despite the increase in the number of data repositories holding tracking data, the vast majority of projects have not been made publicly available [13]. We consider a fixed embargo period with secure data licencing, and a digital object identifier (DOI) for released telemetry datasets, to be the optimum approach to inspire ecologists to move away from a research culture where data is proprietary, while installing measures to protect the intellectual property (IP) of the data owners.

To facilitate data discovery and re-use, open access is also promoted by sharing OzTrack metadata records (i.e. descriptions of the tracking dataset) via the ANDS-RDA portal [45]. RDA is an Internet-based data discovery service that describes the content, context, quality, structure and accessibility of distributed datasets, generated by researchers across Australia. OzTrack generates a metadata record for each project that is compliant with the RIF-CS metadata format. Each metadata record includes the project's title, description, study species, contributors, data licence and embargo period (if relevant) and links to the project's datasets on OzTrack. Metadata records are pushed to RDA via an automatic feed. Additional data regarding biological details (e.g. animal sex, age and body length), hardware specifications (e.g. telemetry device and location schedule) and deployment information (e.g. capture and release location, date and time of deployment and field personnel) may be uploaded in parallel with each location dataset. As telemetry devices have different sources and magnitudes of error [46], and animal 
behaviours can vary according to life history or habitat characteristics [9], providing complete and accurate metadata is critical to ensure that researchers not involved in the data collection process can properly account for accuracy and precision, detection probability and the species' ecology. In many cases, the data collected is not only valuable for the initial study objectives but also valuable for broader, longitudinal comparisons across studies, species, geographical range and years [47]. International projects such as the Starkey Project [48], OBIS-SEAMAP [24] and the European Roe Deer (EURODEER) project [26] illustrate the power of taking a collaborative and synthetic approach across species and localities. Furthermore, increased availability of raw data, and the error estimates for each location, also allow for better parameterizing and scrutinizing of Bayesian and mechanistic models (e.g. $[49,50])$. In these cases, increased sample size enables studies utilising these databases to generate predictive models that are more accurate, more reliable and enable stronger conclusions to be made. However, in order to ensure long-term accessibility and re-use of telemetry data, there is a need for a consensus with regard to best practise and data format standards between researchers, statisticians, tag manufacturers and database developers [15]. Only by creating global standards in data formats and metadata, will it be possible to guarantee compatibility and interoperability between telemetry databases, supporting truly synthetic and trans-disciplinary research.

\section{Future directions}

Testing throughout OzTrack's development highlighted several key areas of improvement [27]. A frequent suggestion from users was the implementation of additional R-based analysis and filtering tools, which have proved informative in the interpretation of animal movement data. While many of these tools were outside the initial scope of our project, the modular system design supports rapid development, implementation and integration within our system architecture [51]. An additional step to further facilitate the development of new tools may be to offer the $\mathrm{R}$ source code to users as an optional output, together with the raw and edited data, and model results (e.g. [8]). This increased transparency would also facilitate the development and implementation of new analysis tools by the wider biotelemetry community.

Another key feature requiring further work is the ability to enrich each location record with the relevant environmental and socio-economic information describing an animal's habitat. The OzTrack system currently holds 21 spatial layers describing climatological, environmental and political information (Additional file 1: Table S1) that with the current version of OzTrack can only be associated with locations on map overlays. Many of these layers are Australian-specific (due to our funding criteria), which allowed us to be more specific on our supplied environmental, climatic and political spatial layers. None of these layers have a temporal component, representing meancomposites of environmental datasets over a particular time period with fixed political boundaries. Furthermore, there is currently no feature to allow users to upload vector- or raster-based spatial objects directly into the system. As animal location data is temporal in nature and animals respond to environmental cues which vary dynamically through time [9], it would be worthwhile incorporating spatial datasets at varying temporal resolutions (e.g. using the MODIS atmosphere, land, cryosphere or ocean data products [52]). OzTrack's Kalman filter tool (obtained from the ukfsst $\mathrm{R}$ package [53]), Movebank's EnvironmentalData Automated Track Annotation (Env-DATA) software [54] and the RNCEP package for R [55] all offer this functionality. The system architecture was structured in a way to allow the animal locations to be linked with spatial datasets at varying temporal resolutions. This new functionality would allow the interactions between animals and their environment to be investigated at a much higher resolution, producing much more meaningful relationships.

\section{Conclusions}

New developments in tracking hardware, pressures for open access to data and the need for multidisciplinary collaborations are accelerating us towards a new age of ecological research [11]. The system infrastructure that we developed provides an integrated, online, free and opensource system that facilitates the uploading, editing, analysis, archiving and sharing of animal tracking datasets. The observed uptake by the Australasian biotelemetry community suggests that the system is proving a valuable resource for researchers despite the stipulation that the data must become publicly available once the embargo period has expired. While the OzTrack system is still in its infancy, our intent is that its framework will promote collaboration, encourage future tool development and help ensure the long-term legacy of animal location datasets. Platform sustainability into the future is however unsubstantiated, due to the short-term nature of funding cycles. Thus, the involvement of the community in terms of funding and system development, as well as integration of the platform into larger eScience infrastructures, may be required to ensure its longevity.

\section{Availability of supporting data}

The datasets supporting the results of this article are available in the OzTrack repository: tracking saltwater crocodiles on the Wenlock River, Cape York, Australia 2013-14 in http://oztrack.org/projects/125; tracking juvenile cassowaries on Cape Tribulation using GPS-based telemetry in https://oztrack.org/projects/2; and habitat restoration 


\section{decisions based on koala movements in http://oztrack.org/ projects $/ 40$.}

\section{Additional files}

Additional file 1: Table S1. Environmental, climatic and political layers currently accessible in OzTrack.

Additional file 2: Table S2. Analysis and filtering tools implemented into the OzTrack system with corresponding $\mathrm{R}$ packages.

\section{Abbreviations}

ANDS: Australian National Data Service; CSV: Comma-Separated Value; DBMS: Database management system; DOI: Digital object identifier; GIS: Geographical information system; IP: Intellectual property; IT: Information technology; KML: Keyhole Markup Language; NeCTAR: National eResearch Collaboration Tools and Resources; OBIS-SEAMAP: Ocean Biogeographic Information System-Spatial Ecological Analysis of Megavertebrate Populations; PNG: Portable Network Graphics; RIF-CS: Registry Interchange FormatCollections and Services; RDA: Research Data Australia; SQL: Structured Query Language; SRS: Spatial reference system; WGS: World Geodetic System; XLS: Microsoft Excel file.

\section{Competing interests}

The OzTrack project was funded by the Australian Government's National eResearch Collaboration Tools and Resources (NeCTAR) eResearch Tools program and the Australian National Data Service (ANDS) Data Capture Program. RGD was partially funded by an Australian Government Endeavour Research Fellowship.

\section{Authors' contributions}

RGD prepared the manuscript, provided the statistical support and developed the underlying R code. CB designed and implemented the spatial database management system, Web Portal, GIS functionality and visualization services. HAC and MW provided the vision and original concept for the OzTrack.org project. All authors contributed to drafting the manuscript. The work was carried out within the laboratories of CEF and JH. All authors read and approved the final manuscript.

\section{Acknowledgements}

We would like to thank our Steering Committee (G. Baxter, M. Hindell, T. Patterson, S. Phinn, C. Simpfendorfer, N. Ward and D. Westcott) and members of the ACEAS Working Group for Advancing the application of animal telemetry data in ecosystem management for ideas that were essential in our system development. We thank S. Fitzgibbon, B. Ellis, G. Grecian, M. Amos, G. Taylor, L. Carpenter, E. Best, B. Arthur, The University of Queensland Conservation Biology 2013/14 class and multiple others who helped test the system and provided helpful feedback. We would also like to acknowledge Peggy Newman for her work during our system's early development. OzTrack was supported through the Australian Government's National eResearch Collaboration Tools and Resources (NeCTAR) eResearch Tools program and the Australian National Data Service (ANDS) Data Capture program. RGD was the recipient of an Australian Government Endeavour Research Fellowship.

\section{Author details}

${ }^{1}$ EcoLab, School of Biological Sciences, The University of Queensland, Brisbane, Australia. ${ }^{2}$ The eResearch Lab, School of ITEE, The University of Queensland, Brisbane, Australia. ${ }^{3}$ Environmental Decisions Group, The University of Queensland, Brisbane, Australia. ${ }^{4}$ Department of Ecosystem Management, School of Environment and Rural Science, The University of New England, Armidale, NSW, Australia.

Received: 22 May 2014 Accepted: 22 December 2014

Published online: 29 January 2015

\section{References}

1. Kenward RE. Historical and practical perspectives. In: Millspaugh JJ, Marzluff JM, editors. Radio tracking and animal populations. CA, USA: Academic Press; 2001. p. 3-12.
2. Tomkiewicz SM, Fuller MR, Kie JG, Bates KK. Global positioning system and associated technologies in animal behaviour and ecological research. Philos Trans R Soc Lond B Biol Sci. 2010;365:2163-76.

3. Eckert SA, Stewart BS. Telemetry and satellite tracking of whale sharks, Rhincodon typus, in the Sea of Cortez, Mexico, and the north Pacific Ocean. Environ Biol Fishes. 2001;60:299-308.

4. Fedak MA, Lovell P, Grant S. Two approaches to compressing and interpreting time-depth information as collected by timed-depth recorders and satellite linked data loggers. Mar Mamm Sci. 2001;17:94-110.

5. Cohn JP. Tracking wildlife. Bioscience. 1999;49:12-7.

6. Ropert-Coudert Y, Wilson RP. Trends and perspectives in animal-attached remote sensing. Front Ecol Environ. 2005;3:437-44.

7. Rutz C, Hays GC. New frontiers in biologging science. Biol Lett. 2009:5:289-92

8. Campbell HA, Watts ME, Dwyer RG, Franklin CE. V-Track: software for analysing and visualising animal movement from acoustic telemetry detections. Mar Freshw Res. 2012;63:815-20.

9. Block BA, Jonsen ID, Jorgensen SJ, Winship AJ, Shaffer SA, Bograd SJ, et al. Tracking apex marine predator movements in a dynamic ocean. Nature. 2011;475:86-90.

10. Wakefield ED, Bodey TW, Bearhop S, Blackburn J, Colhoun K, Davies R, et al. Space partitioning without territoriality in gannets. Science. 2013;341:68-70.

11. Urbano F, Cagnacci F, Calenge C, Dettki H, Cameron A, Neteler M. Wildlife tracking data management: a new vision. Philos Trans R Soc Lond B Biol Sci. 2010;365:2177-85.

12. Boyd IL, Kato A, Ropert-Coudert Y. Bio-logging science: sensing beyond the boundaries. Mem Natl Inst Polar Res, Spec Issue. 2004:58:1-14.

13. Zuckerberg B, Huettmann F, Frair J. Proper data management as a scientific foundation for reliable species distribution modeling. In: Drew CA, Wiersma YF, Huettmann F, editors. Predictive species and habitat modelling in landscape ecology. New York, USA: Springer; 2011

14. Boulton $G$, Campbell $P$, Collins B, Elias $P$, Hall W, Laurie $G$, et al. Science as an open enterprise. The Royal Society Science Policy Centre report 02/12. The Royal Society: London, UK; 2012.

15. Davidson SC. A bigger picture: data standards, interoperability and data sharing. In Spatial database for GPS wildlife tracking data. Edited by Urbano F, Cagnacci F: Springer International Publishing: Switzerland; 2014: 245-270.

16. Cagnacci F, Boitani L, Powell RA, Boyce MS. Animal ecology meets GPSbased radiotelemetry: a perfect storm of opportunities and challenges. Philos Trans R Soc Lond B Biol Sci. 2010;365:2157-62.

17. Coyne MS, Godley BJ. Satellite tracking and analysis tool (STAT): an integrated system for archiving, analyzing and mapping animal tracking data. Mar Ecol Prog Ser. 2005;301:1-7.

18. Huettmann F. Databases and science-based management in the context of wildlife and habitat: toward a certified ISO standard for objective decision-making for the global community by using the internet. J Wildl Manag. 2005;69:466-72.

19. Whalenet [http://whale.wheelock.edu/Welcome.html]

20. Narragansett Bay Coyote Study [http://www.theconservationagency.org/ coyote.htm]

21. Cagnacci F, Urbano F. Managing wildlife: a spatial information system for GPS collars data. Environ Model Software. 2008;23:957-9.

22. Hartog JR, Patterson TA, Hartmann K, Jumppanen P, Cooper S, Bradford R. Developing integrated database systems for the management of electronic tagging data. In Tagging and tracking of marine animals with electronic devices. Volume 9. Edited by J. L. Nielsen, H. Arrizabalaga, N. Fragoso, A. Hobday, Lutcavage M, Sibert J: Springer: Netherlands; 2009: 367-380: Reviews: Methods and Technologies in Fish Biology and Fisheries.

23. Kranstauber B, Cameron A, Weinzerl R, Fountain T, Tilak S, Wikelski M, et al. The Movebank data model for animal tracking. Environ Model Software. 2011;26:834-5.

24. Halpin PN, Read AJ, Fujioka E, Best BD, Donnelly B, Hazen LJ, et al. OBISSEAMAP: the world data center for marine mammal, sea bird and sea turtle distributions. Oceanography. 2009;22:104-15.

25. Wireless Remote Animal Monitoring (WRAM) [http://www.slu.se/WRAM/]

26. Cagnacci F, Focardi S, Hewison AJM, Morellet N, Heurich M, Stache A, et al. Partial migration in roe deer: migratory and resident tactics are end points of a behavioural gradient determined by ecological factors. Oikos. 2011;120:1790-802.

27. Hunter J, Brooking C, Brimblecombe W, Dwyer RG, Campbell HA, Watts ME, Franklin CE: OzTrack-e-infrastructure to support the management, analysis and sharing of animal tracking data. In 9th IEEE International Conference on eScience 22-25 Oct. 2013; Beijing. 2013: 140-147. 
28. OzTrack [http://oztrack.org/]

29. PostgreSQL: The PostgreSQL Global Development Group. 2006. http://www.postgresql.org/.

30. PostGIS: Refractions Research Inc.; 2007. http://postgis.refractions.net/.

31. The Atlas of Living Australia (ALA) Autocomplete Web service [http://api.ala.org.au/]

32. GeoServer [http://geoserver.org/]

33. OzTrack GitHub Website [https://github.com/uq-eresearch/oztrack]

34. R Development Core Team: $\mathrm{R}$ : a language and environment for statistical computing. R Foundation for Statistical Computing 2010. http://www.R-project.org.

35. Urbanek $\mathrm{S}$. Rserve - a fast way to provide R functionality to applications. In Proceedings of the 3rd International Workshop on Distributed Statistical Computing; Vienna, Austria. Edited by Hornik K, Leisch F, Zeileis A. 2003

36. Keitt TH, Bivand R, Pebesma E, Rowlingson B. rgdal: bindings for the geospatial data abstraction library. R Package Version 0.7-8. 2012. http://CRAN.R-project.org/package=rgdal.

37. Spatialreference.org [http://spatialreference.org]

38. Campbell HA, Dwyer RG, Sullivan S, Mead D, Lauridsen G: Chemical immobilization and satellite tagging of free-living southern cassowaries Aust Vet J In Press.

39. Campbell HA, Dwyer RG, Irwin TR, Franklin CE. Home range utilisation and long-range movement of estuarine crocodiles during the breeding and nesting season. PLoS One. 2013;8:e62127.

40. Tufto J, Cavallini P. Should wildlife biologists use free software? Wildl Biol. 2005;11:67-76.

41. Dettki H, Ericsson G, Edenius L. Real-time moose tracking: an internet based mapping application using GPS/GSM-collars. Alces. 2004;40:13-21.

42. Lam $\mathrm{CH}$, Tsontos VM. Integrated management and visualisation of electronic tag data with Tagbase. PLoS One. 2011;6:e21810.

43. Creative Commons Attribution—Non-Commercial-No Derivatives License [http://creativecommons.org/licenses/by-nc-nd/3.0/]

44. Creative Commons Public Domain Mark [http://creativecommons.org/ publicdomain/mark/1.0/]

45. Australian National Data Service (ANDS) Research Data Australia (RDA) Portal [http://researchdata.ands.org.au/]

46. Friar JL, Fieberg J, Hebblewhite M, Cagnacci F, DeCesare NJ, Pedrotti L. Resolving issues of imprecise and habitat-biased locations in ecological analyses using GPS telemetry data. Phil Trans R Soc B Biol Sci. 2010;365:2187-200.

47. Nathan R, Getz WM, Revilla E, Holyoak M, Kadmon R, Saltz D, et al. A movement ecology paradigm for unifying organismal movement research. Proc Natl Acad Sci. 2008;105:19052-9.

48. The Starkey Project [http://www.fs.fed.us/pnw/starkey/]

49. Morales JM, Moorcroft PR, Matthiopoulos J, Frair JL, Kie JG, Powell RA, et al. Building the bridge between animal movement and population dynamics. Philos Trans R Soc Lond B Biol Sci. 2010;365:2289-301.

50. Smouse PE, Focardi S, Moorcroft PR, Kie JG, Forester JD, Morales JM. Stochastic modelling of animal movement. Philos Trans R Soc Lond B Biol Sci. 2010:365:2201-11.

51. van Moorter B. From data management to advanced analytical approaches In Spatial Database for GPS Wildlife Tracking data Edited by Urbano F, Cagnacci F: Springer International Publishing: Switzerland; 2014: 181-212

52. MODIS Web [http://modis.gsfc.nasa.gov/]

53. Lam CH, Nielsen A, Sibert JR. Improving light and temperature based geolocation by unscented Kalman filtering. Fish Res. 2008;91:15-25.

54. Dodge S, Bohrer G, Weinzierl R, Davidson S, Kays R, Douglas D, et al. The environmental-data automated track annotation (Env-DATA) system: linking animal tracks with environmental data. Mov Ecol. 2013;1:3.

55. Kemp M, van Loon E, Shamoun-Baranes J, Bouten W. RNCEP: global weather and climate data at your fingertips. Meth Ecol Evol. 2012:3:65-70.

\section{Submit your next manuscript to BioMed Central and take full advantage of:}

- Convenient online submission

- Thorough peer review

- No space constraints or color figure charges

- Immediate publication on acceptance

- Inclusion in PubMed, CAS, Scopus and Google Scholar

- Research which is freely available for redistribution

Submit your manuscript at www.biomedcentral.com/submit 